\title{
Cubrimiento radicular
}

López-Pinedo M, Li-Wong J. Cubrimiento radicular. Rev Estomatol Herediana. 2011; 21(3):226230.

\section{RESUMEN}

Los procedimientos clínicos de cubrimiento radicular realizados con el fin de tratar las recesiones gingivales, resultan útiles cuando el paciente presenta sensibilidad dentaria, caries radicular o problemas estéticos, como consecuencia de la presencia de recesiones gingivales. Esta revisión pretende brindar una ayuda de consulta definiendo las causas que influyen en la aparición de las recesiones gingivales, así como los diferentes tipos de tratamiento de cubrimiento radicular que podemos brindar a los pacientes.

Palabras clave: RECESIÓN GINGIVAL / SENSIBILIDAD DE LA DENTINA / RAÍZ DEL DIENTE.

\section{Root coverage}

ABSTRACT

The root coverage clinical procedures performed to treat gingival recessions are useful when patients have sensitive teeth, root caries and aesthetics problems, due to the presence of gingival recession. On this review, we provide information on the causes that influence the occurrence of gingival recessions, and different types of treatment for root coverage we can provide to patients.

Key words: GINGIVAL RECESSION / DENTIN SENSITIVITY / TOOTH ROOT.

\section{Martha L. López Pinedo'} Jorge C. Li Wong ${ }^{2}$

'Docente del Departamento Académico de Clínica Estomatológica. Facultad de Estomatología.

Universidad Peruana Cayetano Heredia.

${ }^{2}$ Cirujano-Dentista.

\section{Correspondencia}

Martha López Pinedo

Calle Lloque Yupanqui 1068 - Lima 11, Perú. Teléfono: 993568222

e-mail: martha.lopez@upch.pe

Recibido : 14 de agosto de 2011

Aceptado : 10 de setiembre de 2011

\section{Introducción}

Para tratar el tema de cubrimiento radicular debemos primero empezar por definir recesión gingival. La recesión gingival (RG) es el desplazamiento hacia apical del margen gingival desde su posición a nivel, ó a $1 \mathrm{~mm}$ sobre el límite amelo-cementario (LAC), con exposición de la superficie radicular al medio ambiente oral $(1,2)$.

Desde el punto de vista clínico la RG es medida como la distancia desde el LAC a la extensión más apical del margen gingival (1). La Academia Americana de Periodoncia define a la recesión gingival como la ubicación del margen gingival apical al límite amelocementario (3). Las recesiones son comunes en pacientes con enfermedad periodontal incipiente y también se observa avanzada, en pacientes sin enfermedad periodontal y buena higiene oral. Asimismo se presentan en poblaciones cuya higiene oral es eficiente o deficiente (4). Clínicamente se observa una deformidad mucogingival, con pérdida de inserción; esto ocasiona hipersensibilidad radicular, pobre apariencia estética y lesiones cariosas a nivel cervical de la pieza o piezas dentarias afectadas, por lo que los pacientes llegan a la consulta para preguntar si hay algún tratamiento para mejorar su estética (5).

A lo largo de los años han aparecido numerosas técnicas para corregir las recesiones gingivales como son los procedimientos de colgajo de tejido pediculado y los procedimientos de injerto libre de tejido blando, estos procedimientos han logrado buenos resultados en el mejoramiento de la recesión gingival, aumentando la inserción y altura de la encía (5). Varios estudios clínicos han demostrado un alto porcentaje de éxito usando estos procedimientos, pero el éxito depende de realizar un buen diagnóstico, la localización de la recesión, la técnica a emplear y el entrenamiento del odontólogo (5).

Los profesionales corrigen quirúrgicamente las recesiones gingivales debido a causas estéticas, sensibilidad dentaria, caries o abrasión cervical sin estar necesariamente tratando un problema mucogingival, considerándolo un problema mucogingival cuando existe inflamación y recesión gingival en áreas con poca o nula encía adherida (6).

Uno de los motivos frecuentes en la consulta clínica diaria son las recesiones gingivales y los autores coinciden que su frecuencia aumenta con la edad, en los niños esta frecuencia es aproximadamente del 8\% y en personas mayores de 50 años es hasta del 100\% (5). Es importante identificar el factor etiológico de la recesión gingival antes de realizar el tratamiento propiamente dicho (7).

En esta revisión se pretende describir la etiopatogenia de las recesiones gingivales, los factores predisponentes, los procedimientos empleados para el tratamiento de cubrimiento radicular así como la prevención de las mismas (8) (Fig. 1).

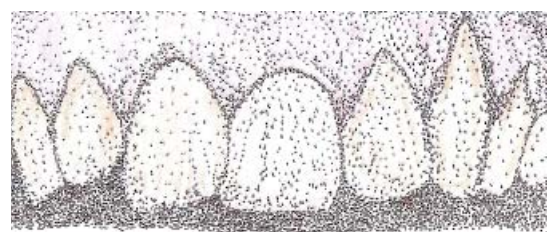

Fig. 1. Piezas 22 y 23 con recesión gingival. Dibujado por: Arq. Denise Goshima Zamami.

\section{Etiología de las recesiones gin- givales}

Existen dos factores causantes de las recesiones gingivales: los predisponentes y los desencadenantes. Los factores predisponentes 
son las condiciones anatómicas y fisiológicas del individuo que podrían influir en el desarrollo de las recesiones gingivales, pero por si solas no podrían ocasionarlo. Los factores desencadenantes son sucesos que iniciaran el desarrollo de la recesión ayudados o no por los factores predisponentes (5,9-12).

\section{Factores predisponentes:}

- Banda de encía queratinizada estrecha o ausente.

- Frenillo de inserción aberrante.

- Malposición dentaria.

- Fenestraciones y dehiscencias alveolares.

Factores desencadenantes:

- Inflamación gingival.

- Cepillado traumático.

- Enfermedad periodontal.

- Obturaciones, prótesis fijas y prótesis removibles mal diseñadas.

Existe evidencia científica para sugerir que la causa principal de las recesiones localizadas en pacientes jóvenes es el cepillado traumático, mientras que en los adultos, la enfermedad periodontal suele ser la causa principal (5).

\section{Clasificación de las recesiones gingivales}

Clasificación de Miller $(1,13)$

Clase I (Fig. 2A):

- Recesión del tejido marginal que no se extiende hasta la unión mucogingival.

- No hay pérdida de hueso ni tejido interdental.

- Se puede pronosticar recuperación del $100 \%$ del cubrimiento radicular.

Clase II (Fig. 2B):

- Recesión del tejido marginal extendida hasta la unión mucogingival o la excede.

- No hay pérdida de hueso o de tejido interdental.
- Se puede anticipar la recuperación del $100 \%$ de cubrimiento radicular.

Clase III (Fig. 2C):

- Recesión del tejido marginal extendida hasta la unión mucogingival o la excede.

- La pérdida de hueso o tejido interdental es leve con respecto al limite amelocementario.

- Se puede anticipar la recuperación del cubrimiento radicular parcial.

Clase IV (Fig. 2D):

- Recesión del tejido marginal se extiende más de la línea mucogingival.

- La pérdida de hueso interdental es de gran importancia.

- No se puede anticipar un cubrimiento radicular.

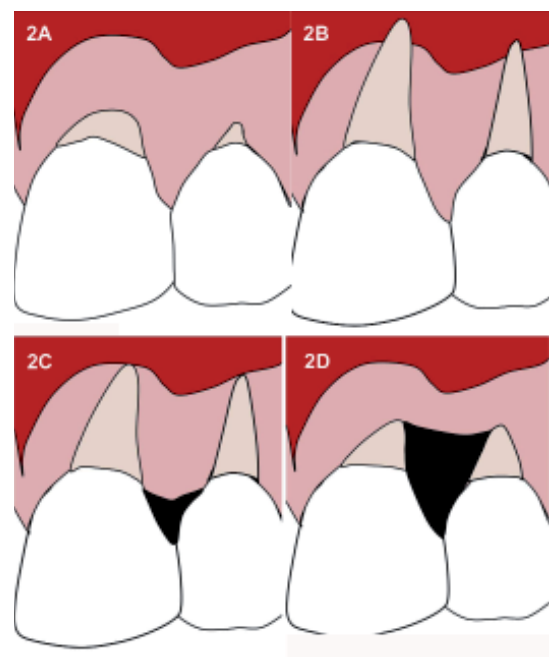

Fig. 2. Clasificasión de Miller para la recesión gingival. 2A. Clase I. 2B. Clase II. 2C. Clase III. 2D. Clase IV. Dibujado por: CD Karina Limaymanta Gonzales.

Indicaciones y contraindicaciones del cubrimiento radicular (14-16).

Indicaciones:

- Recesiones localizadas o generalizadas que ocasionan problemas estéticos.

- Hipersensibilidad radicular.

- Prevención de caries a nivel cer- vical del diente.

- Modificación de la topografía del tejido blando marginal con el propósito de facilitar el control de placa.

Contraindicaciones:

- Pacientes con alteraciones sistémicas.

- Pacientes fumadores.

- Deficiente higiene oral.

Técnicas de cubrimiento radicular

Procedimientos de colgajo pediculado.

1.Colgajo rotacional.

El uso de un colgajo reubicado lateralmente para cubrir áreas con recesión gingival localizada, fue introducida por Grupe y Warren en $1956(5,14,17)$. Esta técnica denominada "operación de colgajo desplazado lateralmente" implicaba el levantamiento de un colgajo de espesor total en una zona donante adyacente al área de la recesión y el desplazamiento lateral de este colgajo para cubrir la superficie radicular expuesta. También se le conoce como "colgajo rotacional oblicuo", "Colgajo rotado" y "colgajo de transposición $(18,19)$ (Fig. 3).

\section{Colgajo de doble papila.}

Cuando el movimiento lateral es hacia mesial o distal del defecto, el colgajo se llama de doble papila (4). Esta indicado esta técnica en recesiones únicas y que adyacente a la recesión se encuentre encía queratinizada de buen grosor y ancho adecuado. El aspecto estético es adecuado. Esta técnica tiene menor porcentaje de necrosis (18, 19) (Fig. 4).

\section{Injertos libres.}

1.Injerto epitelizado.

Este procedimiento fue descrito 

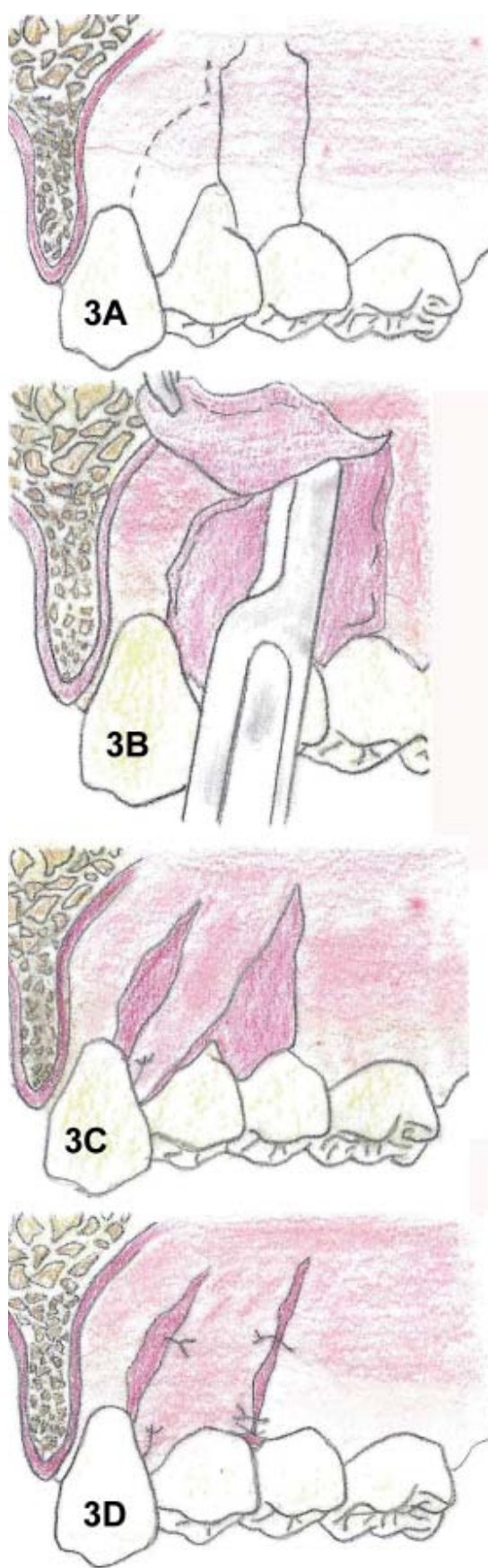

Fig. 3. Colgajo rotacional. 3A. Pieza 24 a la cual se va a realizar el colgajo rotacional (preparación del lecho receptor). 3B. Elevación de un colgajo a nivel de pieza 25. 3C. Colocación de sutura en papila interdental del lecho receptor. 3D. Colocación de sutura simple discontinua para unir tejido transposicionado con tejidos adyacentes. Dibujado por: Ing. Carlos Li Wong.

por Björn en 1963 y sistematizado por Sullivan y Atkins en 1968. La técnica quirúrgica consiste en el reemplazo o aumento de la

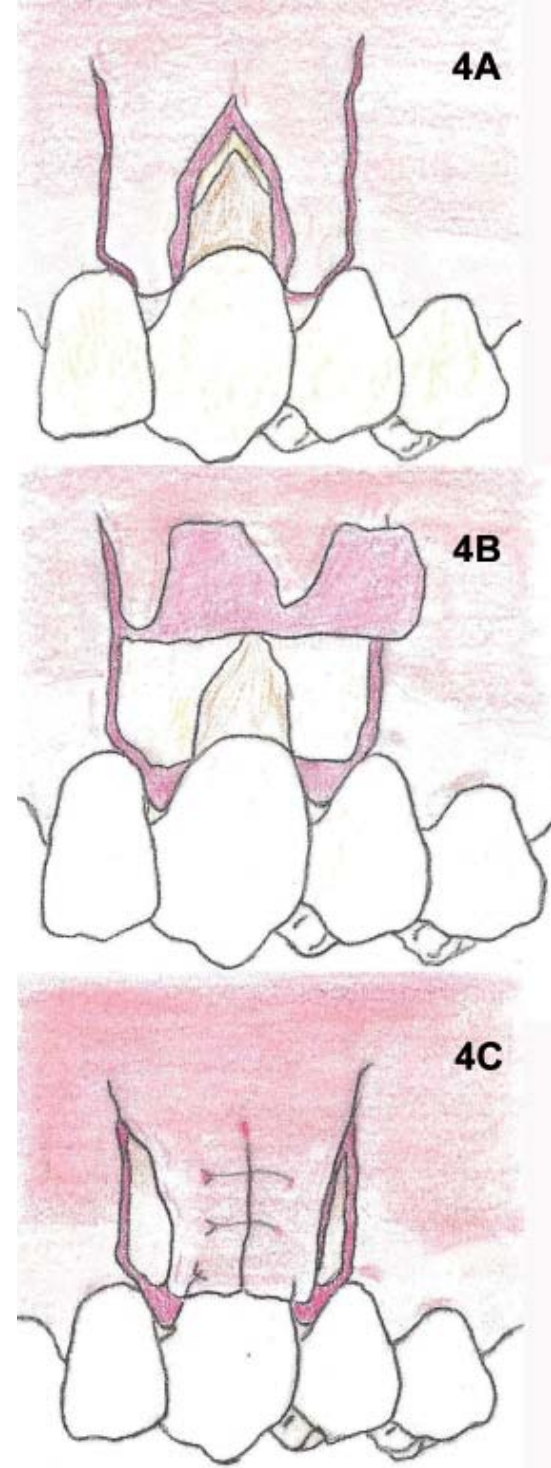

Fig. 4. Colgajo de doble papila. 4A. Pieza 23 con incisiones en forma de "V" a nivel de recesión e incisiones liberantes a distal de pieza 22 y mesial de pieza 24, con aspecto de doble papila. 4B. Elevación de colgajo. 4C. Sutura de la doble papila, previa colocación del colgajo a nivel del límite amelocementario. Dibujado por: Ing. Carlos Li Wong.

mucosa móvil no queratinizada por tejidos epitelizados o encía queratinizada "verdadera" que se transfiere usualmente del paladar duro. El compromiso estético es uno de los factores que contraindican su aplicación, ya que debe usarse en áreas no visibles cuando el paciente habla o sonríe $(5,8,14)$.
2.Injerto de tejido conectivo subepitelial.

Esta técnica obtiene el injerto a nivel del paladar duro o de la almohadilla retromolar, transfiriendo el tejido conectivo subepitelial y llevándolo hasta la zona receptora o zona de la recesión gingival. El injerto de tejido conectivo es preferible al injerto epitelizado porque la herida palatina producida es menos invasiva y el resultado estético en la zona receptora es mejor $(15,20)$.

3.Injerto de tejido conectivo subepitelial cubierto por un colgajo avanzado coronalmente.

Es el "gold estándard" de los tratamientos de cubrimiento radicular, el injerto se coloca directamente sobre la raíz expuesta, movilizándose un colgajo el cual se dejará en posición coronaria, con el propósito de cubrir el injerto. Entre las ventajas de esta técnica son las pocas molestias que experimenta el paciente en la zona dadora (paladar) y los buenos resultados estéticos (encía color rosado queratinizado) obtenidos en la zona receptora. La principal desventaja es que es una técnica difícil, requiere entrenamiento del odontológo (5,21-24) (Fig. 4).

\section{Factores de éxito para el cubri- miento radicular}

Relacionados con el paciente.

La mala higiene oral después de la intervención influye negativamente en el cubrimiento radicular. Así mismo el factor causal predominante es el desarrollo de las recesiones gingivales en el cepillado traumático y por eso debe corregirse este factor para obtener un resultado óptimo (5).

La influencia del tabaco es hasta ahora un tema polémico ya que algunos estudios informan que el 


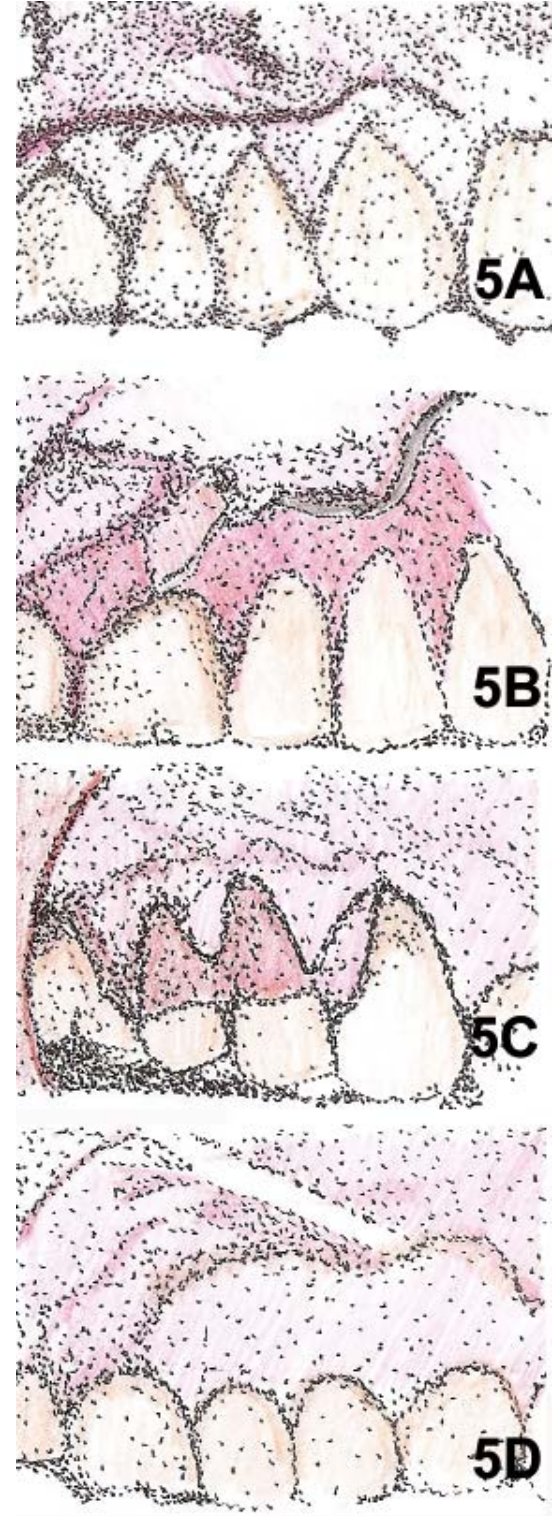

Fig. 5. Injerto de tejido conectivo. 5A. Recesiones gingivales en piezas 13, 14 y 15. 5B. Elevación de colgajo a nivel de recesiones. 5C. Colocación de in jerto conectivo a nivel de recesiones. 5D. Resultado final del Tratamiento. Dibujado por: Arq. Denise Goshima Zamami.

fumar produce resultados menos favorables en el cubrimiento radicular (19).

Zucchelli et al. (25) estableció que la expectativa de cubrimiento radicular disminuía en $0,52 \mathrm{~mm}$ en pacientes fumadores. Mientras que otros estudios no mostraron diferencias entre fumadores y no fumadores (16).

\section{Relacionados con el sitio.}

Entre los factores relacionados al sitio, el soporte periodontal interdental puede ser el de mayor importancia para los resultados del cubrimiento radicular. En las recesiones clases I y II de Miller el cubrimiento radicular es completo, mientras que en las clases III y IV de Miller el cubrimiento es parcial por la pérdida del soporte periodontal (22).

Relacionados con la técnica quirúrgica.

Baldi et al. (23) comprobaron una asociación positiva entre la reducción de la recesión y el espesor del colgajo. En las clases I y II de Miller el cubrimiento radicular completo solo se consiguió cuando el espesor del colgajo era de 0,8 $\mathrm{mm}$. Sin embargo el resultado del tratamiento de cubrimiento radicular es indistinto si se usa un colgajo a grosor total o parcial.

Un factor importante para PiniPrato et al. (24) en el resultado del colgajo avanzado o desplazado coronalmente es la tensión del colgajo, los mejores resultados se obtienen cuando el colgajo se adapta pasivamente sobre la superficie radicular.

\section{Conclusiones}

- El cubrimiento radicular es un método conveniente para tratar la sensibilidad dentaria.

- Los factores desencadenantes deben ser controlados antes de iniciar el tratamiento de cubrimiento radicular, ya que estos influyen en la aparición de las recesiones gingivales.

- El hábito de fumar influye negativamente en el éxito del tratamiento de cubrimiento radicular.

- En recesiones clases I y II de Miller, el tratamiento es éxitoso a comparación de las clases III y IV de Miller.

- El tratamiento de elección para realizar el cubrimiento radicular es el injerto de tejido conectivo subepitelial acompañado de un colgajo desplazado coronalmente (Gold Standard).

\section{Referencias bibliográficas}

1. Zucchelli G, Mele M, Stefanini M, Mazzotti C, Mounssif I, Marzadori M, Montebugnoli L. Predetermination of root coverage. J Periodontol. 2010; 81(7):1019-26.

2. Haghighat K. Modified semilunar coronally advanced flap. J Periodontol. 2006; 77(7):12749.

3. Glossary of Periodontal Terms. American Academy of Periodontology. 4 ed. Chicago; 2001.

4. De Rossi. Atlas de odontología restauradora y periodoncia. 2 ed. Bogota: Editorial Panamericana; 2003.

5. Lindhe J. Periodontología Clínica. 3 ed. Buenos Aires: Editorial Panamericana; 2005.

6. Maynard JG Jr, Wilson RD. Physiologic dimensions of the periodontium significant to the restorative dentist. J Periodontol. 1979; 50(4):170-4.

7. Cabral Cruz AC, Barquero Cordero E, Sartori R, Pilatti GL, Santos FA. Recubrimiento de las recesiones gingivales con la técnica de injerto conjuntivo subepitelial asociado al deslizamiento coronario del colgajo: relato de caso clínico. Acta Odontol Venez. 2008; 46(3):33741.

8. Carranza F. Periodontologia clínica. 9 ed. México: Editorial Interamericana; 2004.

9. Malla E. Prótesis fija estética: Enfoque clínico e interdiscipli- 
nario. 1 ed. Madrid: Elsevier; 2007.

10.Cavenaghi G, Caccianiga GL, Baldoni M, Lamedica M. Comparación entre técnicas bilaminares y regenerativas para recubrir las raíces. Av Periodon Implantol. 2000; 12(3):127-136.

11. Kao RT, Fagan MC, Conte GJ. Thick vs. thin gingival biotypes: a key determinant in treatment planning for dental implants. J Calif Dent Assoc. 2008; 36(3):193-8.

12.Rocha Abramovich A, Ferrus Cruz J. Tratamiento de recesiones múltiples localizadas: a propósito un caso. Av Periodon Implantol. 2007; 19(1):19-28.

13. Miller PD Jr. A classification of marginal tissue recession. Int $\mathrm{J}$ Periodontics Restorative Dent. 1985; 5(2):8-13.

14.Rateitschake E, Rateitschake K, Wolf H, Hassell T. Periodontology: Color atlas of dental medicine. 3th ed. New York: Thieme; 2005.

15. Camargo PM, Melnick PR, Kenney EB. The use of free gingival grafts for aesthetic purposes.
Periodontol 2000. 2001; 27:7296.

16.Trombelli L, Scabbia A. Healing response of gingival recession defects following guided tissue regeneration procedures in smokers and non-smokers. J Clin Periodontol. 1997; 24(8):52933.

17.Kassab MM, Cohen RE. Treatment of gingival recession. J Am Dent Assoc. 2002; 133(11):1499-506.

18.Bouchard P, Malet J, Borghetti A. Decision-making in aesthetics: root coverage revisited. Periodontol 2000. 2001; 27:97120.

19. Sato N. Cirugía Periodontal: Atlas Clínico. 2 ed. Bogota: Quintessence; 1997.

20.Langer B, Langer L. Subepithelial connective tissue graft technique for root coverage. J Periodontol. 1985; 56(12):71520.

21.Caffesse RG, Guinard EA. Treatment of localized gingival recessions. Part II. Coronally repositioned flap with a free gingival graft. J Periodontol. 1978;
49(7):357-61.

22. Miller PD Jr. Root coverage with the free gingival graft. Factors associated with incomplete coverage. J Periodontol. 1987; 58(10):674-81.

23.Baldi C, Pini-Prato G, Pagliaro U, Nieri M, Saletta D, Muzzi L, Cortellini P. Coronally advanced flap procedure for root coverage. Is flap thickness a relevant predictor to achieve root coverage? A 19-case series. J Periodontol. 1999; 70(9):1077-84.

24.Pini Prato G, Pagliaro U, Baldi C, Nieri M, Saletta D, Cairo F, Cortellini P. Coronally advanced flap procedure for root coverage. Flap with tension versus flap without tension: a randomized controlled clinical study. J Periodontol. 2000; 71(2):188-201.

25.Zucchelli G, Clauser C, De Sanctis M, Calandriello M. Mucogingival versus guided tissue regeneration procedures in the treatment of deep recession type defects. J Periodontol. 1998; 69(2):138-45. 